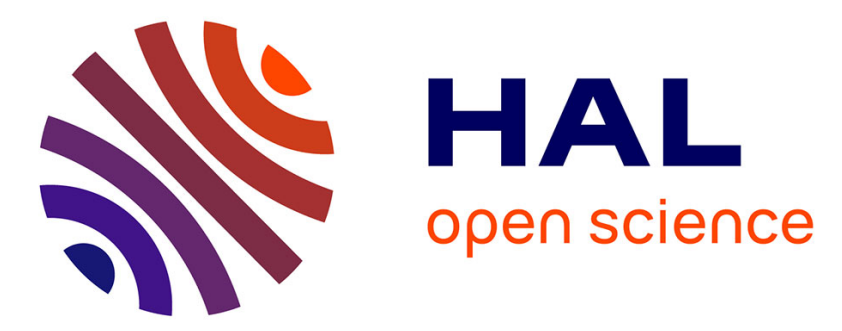

\title{
Microstructure and concentration fluctuations in alcohol-Toluene and alcohol-Cyclohexane binary liquids: A small angle neutron scattering study
}

\author{
Ramona Mhanna, R. Lefort, Laurence Noirez, D. Morineau
}

\section{- To cite this version:}

Ramona Mhanna, R. Lefort, Laurence Noirez, D. Morineau. Microstructure and concentration fluctuations in alcohol-Toluene and alcohol-Cyclohexane binary liquids: A small angle neutron scattering study. Journal of Molecular Liquids, 2016, 218, pp.198-207. 10.1016/j.molliq.2016.02.070 . hal01283902

\section{HAL Id: hal-01283902 \\ https://hal.science/hal-01283902}

Submitted on 7 Mar 2016

HAL is a multi-disciplinary open access archive for the deposit and dissemination of scientific research documents, whether they are published or not. The documents may come from teaching and research institutions in France or abroad, or from public or private research centers.
L'archive ouverte pluridisciplinaire HAL, est destinée au dépôt et à la diffusion de documents scientifiques de niveau recherche, publiés ou non, émanant des établissements d'enseignement et de recherche français ou étrangers, des laboratoires publics ou privés. 


\title{
Microstructure and concentration fluctuations in alcohol-Toluene and alcohol-Cyclohexane binary liquids: A small angle neutron scattering study
}

\author{
R. Mhanna ${ }^{\mathrm{a}, \mathrm{b}}$, R. Lefort ${ }^{\mathrm{a}}$, L. Noirez ${ }^{\mathrm{c}}$, D. Morineau ${ }^{\mathrm{a},{ }^{*}}$ \\ ${ }^{a}$ Institute of Physics of Rennes, CNRS-University of Rennes 1, UMR 6251, F-35042 Rennes, \\ France. \\ ${ }^{\mathrm{b}}$ Institut Laue-Langevin, 71 avenue des Martyrs, F-38000 Grenoble, France \\ ${ }^{\mathrm{c}}$ Laboratoire Léon Brillouin (CEA-CNRS), Paris-Saclay University, F-91191 Gif-sur-Yvette, \\ France
}

\section{ABSTRACT:}

The origin of concentration fluctuations in a series of binary liquids is examined by small angle neutron scattering in relation with H-bonded micellar clusters and the aggregation of alcoholrich domains, which is also related to the well-known observation of a prepeak in diffraction spectra of alcohols. The results suggest that concentration fluctuations do not arise from size variation and re-arrangement of the mesoscopic domains but from the micellar-clusters themselves. We evaluate the scattering intensity at small angles and deduce the OrnsteinZernike correlation lengths, the Bhatia-Thornton concentration fluctuations and the KirkwoodBuff integrals. An alternative approach, based on the Guinier approximation was applied, indicating that the observed inhomogeneity could be related to spherical particles with diameter comparable to the H-bonded multimers. We compare the structure factor of different systems when varying the molecular interactions: the alcohol-solvent interaction has been tuned with Toluene and Cyclohexane aprotic solvents, and the amphiphilic character of the alcohol, by going from Tert-butanol to Ethanol and Methanol.

\footnotetext{
* Corresponding author.

E-mail address:denis.morineau@univ-rennes1.fr
} 


\section{Introduction}

Many binary liquids containing alcohol molecules display a strong deviation from the ideal mixture behavior. This deviation can be attributed to the incomplete mixing of the distinct interacting molecules at the microscopic level even though these liquids are often macroscopically miscible $[1,2,3,4]$. These original features are often observed for small-chains alcohols, also denoted hydrotropes, which exhibit an amphiphilic character but are too small to form ordinary micelles in solution [5]. Recent studies have linked the structural complexity of aqueous solutions of small H-bonded molecules to the interplay between concentration fluctuations and self-aggregation, the concept of molecular emulsions being introduced by Kezic and Perera $[6,7,8,9]$. It turns out that multiscale approaches are required for the structural characterization of $\mathrm{H}$-bonded micelle-like clusters and their spatial organization during dilution in water or in an aprotic solvent, the latter acting as H-bond diluent, which modulates the molecular interactions $[10,11,12,13,14]$.

Water - Tert-butanol (TBA) mixture is one of the systems that are the most debated currently, being considered as the prototypical system to advance our understanding of hydrotrope solubility in aqueous solution $[5,8,9,15,16,17,18,19]$. Analogous interest arises from studies on non-aqueous solvent - TBA mixtures. Distinct self-association and segregation processes have been examined in detail for Tert-butanol (TBA) - Toluene (TOL) and Tertbutanol (TBA) - Methylcyclohexane (MCY) systems [10]. For this purpose, two techniques were applied: Raman spectroscopy to quantify the size distribution of the H-bonded micellar multimeric clusters and neutron diffraction to investigate the spatial correlation between these clusters at larger distances [10]. According to the analysis of Raman results, TBA molecules mostly form H-bonded tetrameric units, which exhibit an exceptional stability [20,21]. They persist under conditions of high dilution, i.e. molar fraction $x$ of TBA as low as $x=0.1$ in TBAMCY mixtures. On the contrary, the spatial correlations between these neighboring micellar clusters seem much more sensitive to dilution. This phenomenon was revealed in the neutron diffraction structure factor by the suppression of a pre-peak, located at a momentum transfer $q \mathrm{PP}=0.7 \AA^{-1}$, which is a well-known signature of TBA intermediate range order and the existence of correlations between H-bonded units [10,22,23,24,25,26,27,28,29,30]. It was shown that the addition of a small amount of aprotic solvent is sufficient to turn off the prepeak intensity with a complete extinction for $x<0.6-0.8$ for TBA-TOL and TBA-MCY [10]. Accordingly, it was suggested that the microstructure of these mixtures could be expressed in terms of noninteracting micellar TBA clusters for a broad range of compositions, while correlated clusters 
and diluted monomers were prevailing for high and low values of $x$ respectively (cf. Fig. 12 in ref. [10]).

In this article, we extend the study of the segregation phenomenon towards a lower $q$ region. Small angle neutron scattering (SANS) is a complementary method to address the inhomogeneity of the mixtures in terms of concentration fluctuations, which are signatures of single-component-rich domains triggered by selective molecular interactions. Different hydrogenated/deuterated isotopic compositions are used to vary the scattering length density of the components of the binary liquids, and optimize the contrast between domains of different compositions. The characteristic correlation length of the inhomogeneities has been determined using the Ornstein-Zernike model [31]. An alternative approach, based on the Guinier approximation was applied, indicating that the observed inhomogeneity could be expressed in terms of weakly interacting spherical particles with diameter comparable to the H-bonded multimers [32]. The formalism of Bhatia and Thornton has been applied to decouple the different correlation functions related to density and concentration from the total neutron structure factor at zero- $q$ [33]. The fluctuations of the local composition have been quantified by the evaluation of the Kirwood-Buff integrals (KBI), which can be determined from the experimental forward scattering intensities [34].

The aim of this study is to shed light on the different phenomena, expressed in terms of self-association, multimers and concentration fluctuations that contribute to the complex microstructure of prototypical alcohols mixed with aprotic solvents. Different systems have been considered to vary their molecular interactions. First, the alcohol-solvent interaction has been tuned by comparing TBA-TOL and TBA-Cyclohexane (TBA-CYC) mixtures. The amphiphilic character of the alcohol has been then varied, going from TBA, to Ethanol (ETA) and Methanol (MEA). The overall assessment suggests that concentration fluctuations are primarily determined by the existence of H-bonded micellar-clusters with weak inter-cluster interaction rather than from fluctuations of the size and arrangement of mesoscopic alcoholrich domains.

\section{Experimental}

Small-angle neutron scattering experiments were performed on the PAXY diffractometer (LLB, Saclay). The sample detector distance chosen was $1.6 \mathrm{~m}$ and the neutron wavelength used was $3.5 \AA$ allowing the measurement of the diffracted intensity in a momentum transfer range $\left(0.04<q<0.5 \AA^{-1}\right)$. The samples were prepared from hydrogenated Tert-butanol 
TBA(H) mixed with deuterated Toluene TOL(D) or with Cyclohexane CYC(D) as well as their opposite isotopic compositions: the four types of mixtures are latter denoted TBA(H)-TOL(D), TBA(H)-CYC(D), TBA(D)-TOL(H) and TBA(D)-CYC(H), respectively. Methanol and Ethanol hydrogenated samples were also prepared with deuterated Toluene i.e. MEA(H)TOL(D) and ETA(H)-TOL(D) mixtures. The bulk samples were prepared in volume fraction covering the concentration range $x=0-1$ ( $x$ being the volume fraction of the alcohol). The measurements were conducted at room temperature with a thermal bath regulator set to $22^{\circ} \mathrm{C}$. The solutions were prepared in advance so the mixtures are thermodynamically at equilibrium. The solutions were filled in $1 \mathrm{~mm}$ quartz Hellma cells. The intensities were corrected from empty cell contribution by subtraction of the filled and empty samples spectra, divided by their own measured forward transmission. The correction of the systematic variation of the detector efficiency and normalization of $I(q)$ to absolute unit were performed with respect to the measured intensity of hydrogenated water filled in the same cell, having a flat signal with a known value of its incoherent cross section $\left(\Sigma\left(\mathrm{H}_{2} \mathrm{O}\right) / 4 \pi=0.445 \mathrm{~cm}^{-1}\right)$.

\section{Tert-butanol-Toluene and Tert-butanol-Cyclohexane systems}

\subsection{Experimental Results}

The neutron scattering profile shows an increase of the scattering intensity at low $q$ for all the binary mixtures (Figs. 1 and 2), which firmly indicates that the molecular distribution in the liquid is heterogeneous. In the $q$-range covered by the SANS instrument, albeit a prominent maximum intensity centered at $q=0$, the scattered intensity does not exhibit any measurable peak at finite $q$-value.

Teubner and Strey have introduced a phenomenological approach to interpret the scattering intensity of microemulsions [35]. The theory has been later discussed for aqueous solutions of short-chains amphiphilic molecules, including TBA and discussed in the frame of so-called molecular emulsions $[6,7,8,9,36]$. According to Teubner-Strey approach, the scattering function is expressed as the inverse four-order polynomial in $q$. The microstructure is defined by two characteristic lengths corresponding to the domain size $d$ (i.e. periodic repeat distance) and the correlation length $\xi$. A distinct prediction of this theory is the appearance of a prepeak located at $q=2 \pi / d$, that reflects the pseudo-periodic organization of the domains. The absence of a diffraction peak in the present SANS measurement suggests that on the length scale probed there is no observable microstructure with pseudo periodic order. We remind that the correlations between micellar clusters show up as a prepeak at $q=0.7 \AA^{-1}$ which is then related 
to smaller (near-molecular) repeat distances. In addition, it was shown that this prepeak rapidly vanishes with dilution, which is at variance with the small angle scattering intensity that readily increases [10]. This suggests that the intercluster correlations are effectively lost by dilution. The Debye-Bueche model is an alternative model which is often used to describe phase separated binary systems [31]. The scattering intensity is a squared Lorentzian function $I(q) \propto$ $\frac{1}{\left(1+q^{2} \xi^{2}\right)^{2}}$ introducing a unique correlation length $\xi$. It is indeed a special case of the TeubnerStrey model for $\xi \ll d$. In practice, it was observed in other studies that this is reduced to an Ornstein-Zernike function (single Lorentzian form) if the four-order term $(q \xi)^{4}$ is not detectable, which turns out to be the case in the present study, given that $q \xi<1$ [37]. This is analyzed quantitatively in the following.
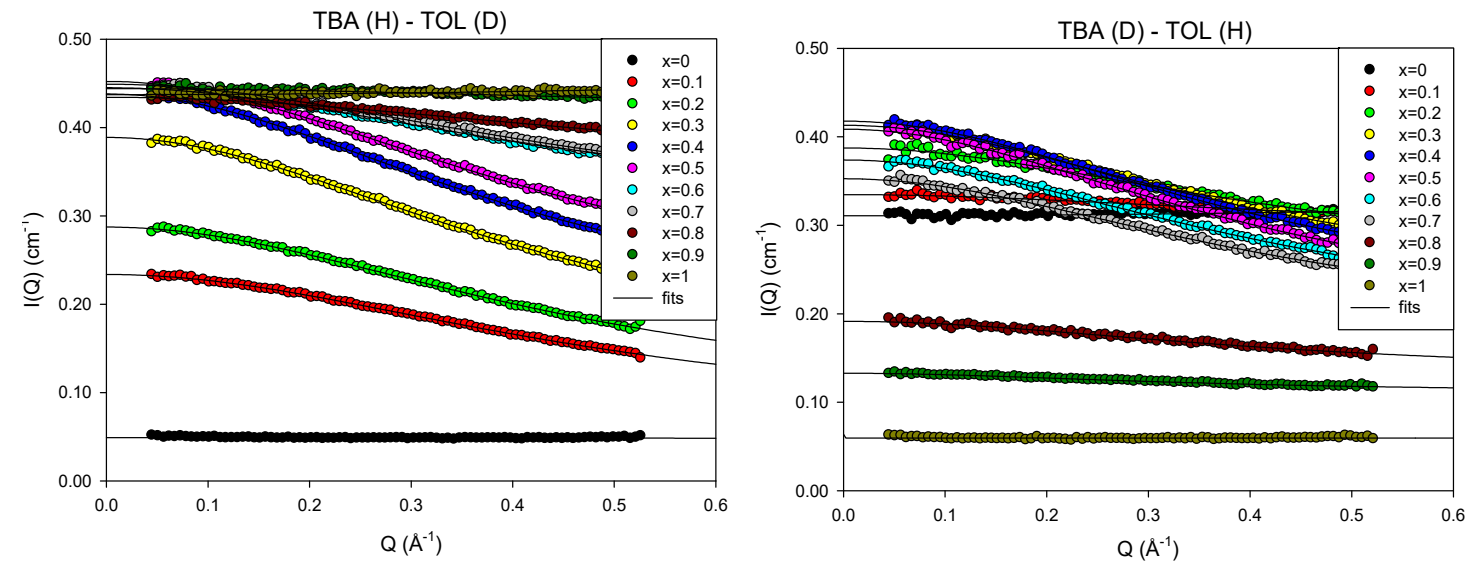

Fig. 1. SANS structure factors of Tert-butanol-Toluene mixtures, comprising hydrogenated TBA with deuterated TOL (left panel) and vice-versa (right panel). The different curves correspond to eleven values of the TBA volume fractions $x$ from 0 to 1 . The plotted solid lines are fits to the Ornstein-Zernike model. 

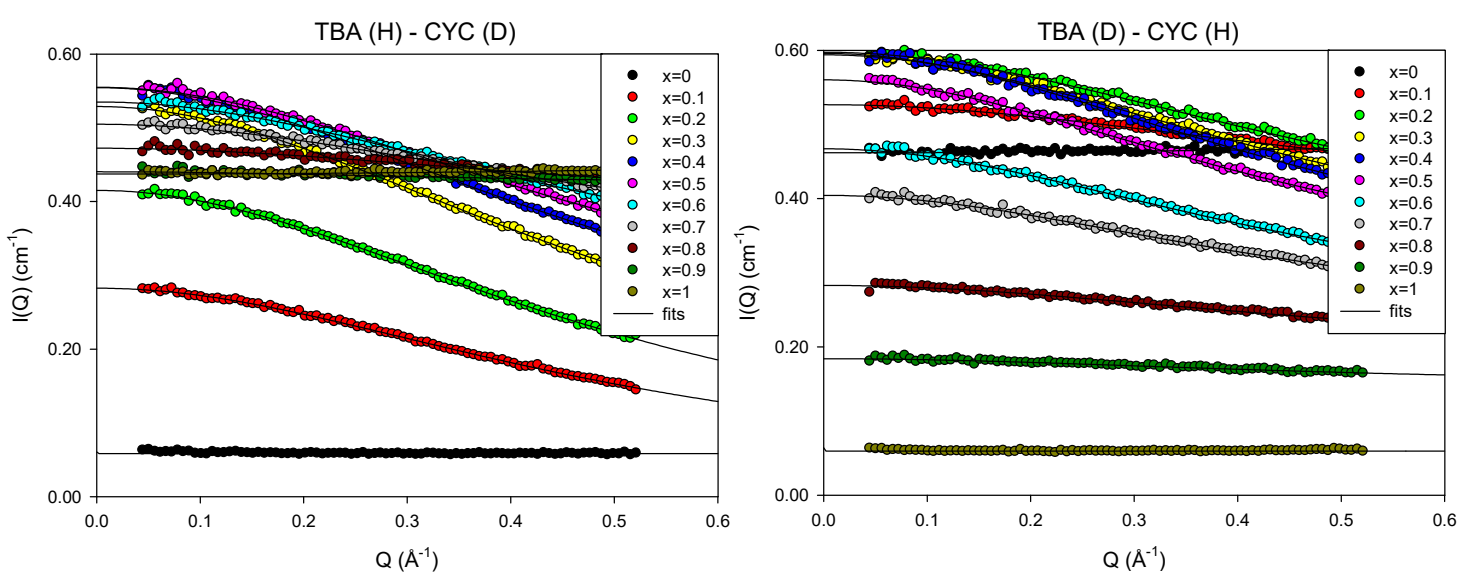

Fig. 2. SANS structure factors of Tert-butanol-cylcohexane mixtures, comprising hydrogenated TBA with deuterated CYC (left panel) and vice-versa (right panel). The different curves correspond to eleven values of the TBA volume fractions $\mathrm{x}$ from 0 to 1 . The plotted solid lines are fits to the Ornstein-Zernike model.

\subsection{Ornstein-Zernike Analysis}

The scattered intensity was modelled by a Lorentzian function according to the OrnsteinZernike theory [38]. Extending the case of phase separating systems [39,40], this formalism is also known to accurately reproduce the scattering intensity of many non-ideal binary mixtures $[41,42,43]$ and can be defined as:

$I(\mathrm{q})=\frac{A}{1+q^{2} \xi^{2}}+B$

where the first term comprises the $q$-dependent coherent scattering, the second term represents the $q$-independent background and $\xi$ is the correlation length characterizing the spatial extension of the scattering length density fluctuations. This formalism can be applied to describe the scattering intensity at zero angle which can originate from domains with distinct scattering length density caused by density and/or concentration fluctuations. Experimentally, the coherent forward scattering intensity $I_{\mathrm{coh}}(0)$ can be obtained via extrapolation to zero- $q$. The first term $A$ arises from the $q$-dependent coherent scattering. The second term $B$ comprises the coherent scattering arising from the isothermal compressibility and the incoherent scattering, which do not depend on $q$, according to 


$$
\begin{aligned}
& A=I_{\text {coh }}(0)-\rho^{2} k_{B} T \kappa_{T} \bar{b}^{2} \approx I_{\text {coh }}(0) \\
& B=\frac{\sigma_{i n c}}{4 \pi}+\rho^{2} k_{B} T \kappa_{T} \bar{b}^{2} \approx \frac{\sigma_{i n c}}{4 \pi}
\end{aligned}
$$

where $\rho, k_{B}, T, \kappa_{T}, \sigma_{\text {inc }}$ are the molecules number density, the Boltzmann constant, the temperature, the isothermal compressibility, and the average incoherent cross section per volume, respectively. The average molecular scattering length $\bar{b}$ is defined as $\bar{b}=x_{1} b_{1}+(1-$ $\left.x_{1}\right) b_{2}$, where $x_{1}$ is the aprotic solvent molar fraction, $b_{1}$ and $b_{2}$ are the sums of the coherent scattering lengths of the atoms constituting the aprotic and the alcohol molecules. The isothermal compressibility of the studied liquids being around $910^{-10} \mathrm{~Pa}^{-1}$, its contribution to the scattered intensity is very small compared to $A$ and $B$ for the studied mixtures compositions. For instance, it varies from $3.710^{-5} \mathrm{~cm}^{-1}$ (resp. $3.410^{-4} \mathrm{~cm}^{-1}$ ) for hydrogenated TBA (resp. TOL) to $1.510^{-2} \mathrm{~cm}^{-1}$ (resp. $1.210^{-2} \mathrm{~cm}^{-1}$ ) for deuterated TBA (resp. TOL).

A fit of the coherent intensity with an Ornstein-Zernike type model was performed and plotted as solid lines in Figs. 1 and 2. The resulting fit parameters of the Lorentzian (correlation length, amplitude and background) for the different samples are plotted in Figs. 3 and 4. The correlation length seems to remain almost constant for all mixture compositions and all the samples, with values close to the molecular scale (about $2 \AA$ ). The background increases almost linearly as expected with increasing Hydrogen content due to incoherent scattering, in agreement with the calculated values of $\frac{\sigma_{i n c}}{4 \pi}$ shown as dashed lines. Regarding the amplitude, the general trend is characterized by a maximum reached around the intermediate compositions. The vanishing values obtained for the pure liquids are consistent with the absence of heterogeneities in the scattering length densities in these cases. 

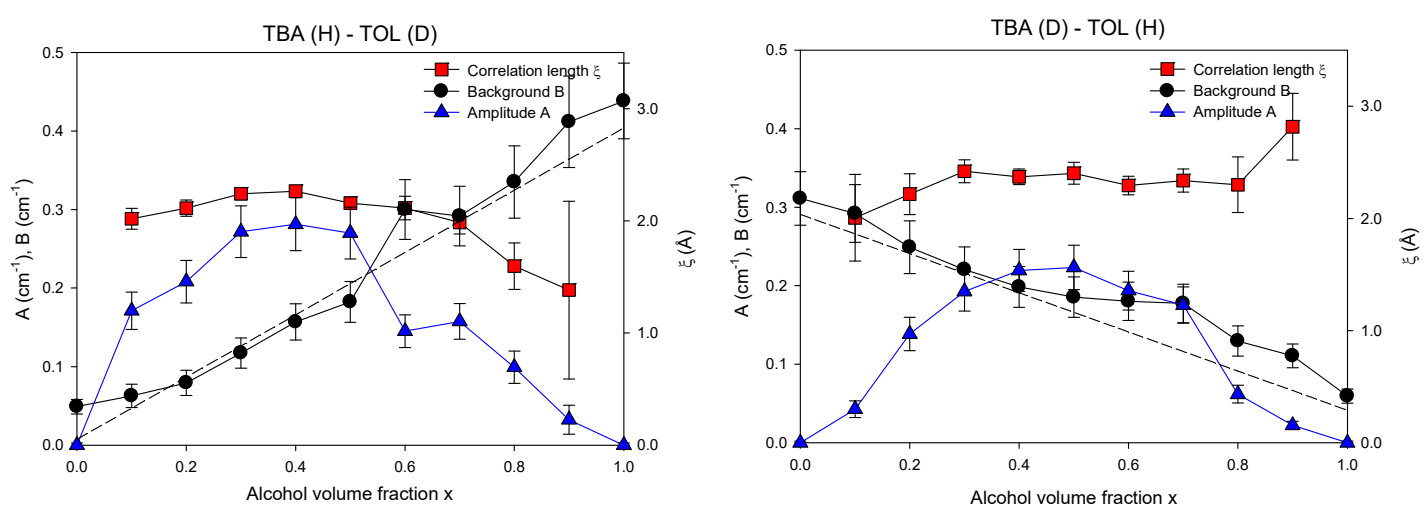

Fig. 3. Parameters (amplitude, correlation length and background) obtained from a fit with the Ornstein-Zernike model of the SANS structure factors of Tert-butanol-Toluene mixtures, comprising hydrogenated TBA with deuterated TOL (left panel) and vice-versa (right panel).
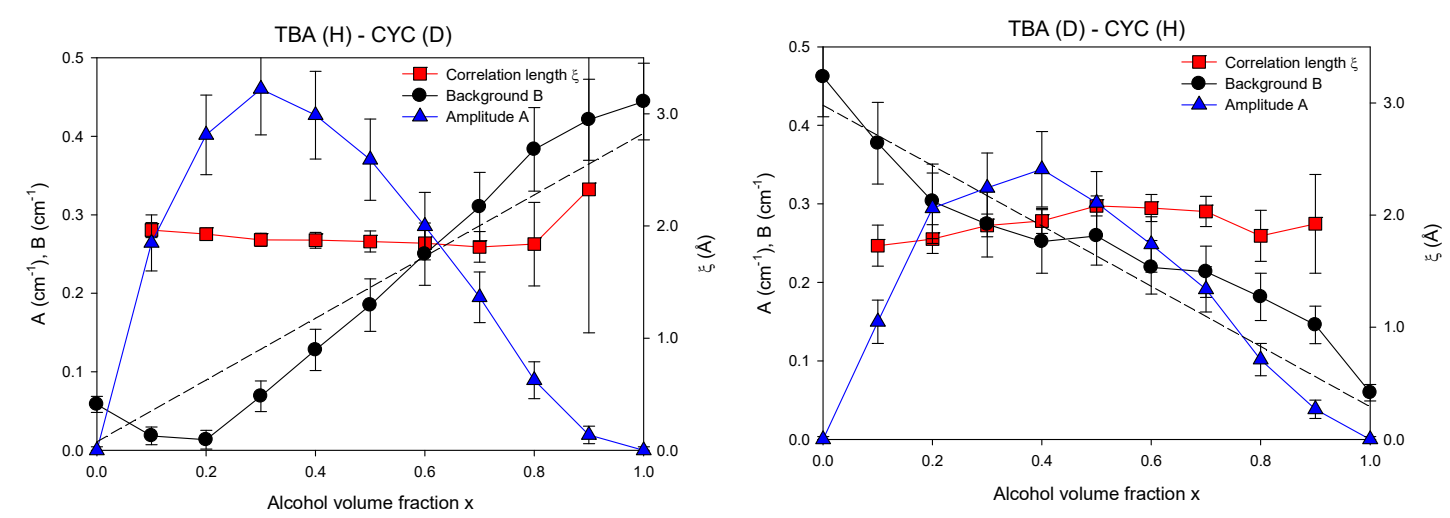

Fig. 4. Parameters (amplitude, correlation length and background) obtained from a fit with the Ornstein-Zernike model of the SANS structure factors of Tert-butanol-Cyclohexane mixtures, comprising hydrogenated TBA with deuterated TOL (left panel) and vice-versa (right panel).

\subsection{Bhatia-Thornton analysis}

Following Bhatia and Thornton the zero angle coherent scattering of a binary mixture can be expressed as a linear combination of distinct fluctuation terms [33]:

$\frac{I_{\mathrm{coh}}(0)}{\rho}=\bar{b}^{2} S_{N N}(0)+\left(b_{1}-b_{2}\right)^{2} S_{C C}(0)+2 \bar{b}\left(b_{1}-b_{2}\right) S_{N C}(0)$ 
$S_{C C}(0)$ represents the fluctuations in the concentration of one molecular type, $S_{N N}(0)$ describes the fluctuations in the molecular number and $S_{N C}(0)$ represents the correlation between the two types of fluctuations. The latter terms are linked to the $S_{C C}(0)$ at zero limit of the scattering intensity with the following relations:

$S_{N N}(0)=\rho k_{B} T \kappa_{T}+\delta^{2} S_{C C}(0) \approx \delta^{2} S_{C C}(0)$

$S_{N C}(0)=-\delta S_{C C}(0)$

where $\delta=\rho\left(v_{l}-v_{2}\right)$ is the dilatation factor and $v_{l}, v_{2}$ the partial molar volumes per molecule of the two components.

The slight dependence of $v_{1}$ and $v_{2}$ on the mixture composition, which arises from the excess volume was neglected in our calculation. We checked the weak influence of this assumption for the mixtures and compositions where these excess quantities were available. For instance, in the case of TBA-TOL mixtures, the excess volume found in the literature is $V_{\text {excess }}<0.5$ $\mathrm{cc} / \mathrm{mol}$ and the molar volumes of TBA and TOL are 95 and $105 \mathrm{cc} / \mathrm{mol}$ respectively (i.e., $\Delta V=10 \mathrm{cc} / \mathrm{mol}$ ) [44]. This result in an excess volume which is less than $5 \%$ of $\Delta V$, which is within the error bars of our measurements.

Furthermore, $S_{C C}(0)$ can be calculated from the forward coherent scattering using the following equation, which is obtained after insertion of Eqs. (5) and (6) into Eq. (4):

$I_{\mathrm{coh}}(0)=\rho^{2} k_{B} T \kappa_{T} \cdot \bar{b}^{2}+\rho\left[\bar{b} \delta-\left(b_{1}-b_{2}\right)\right]^{2} S_{C C}(0)$

The fluctuation terms in the solutions were obtained from the amplitude of the Lorentzian fit combining Eqs. (3) and (7), which gives:

$S_{C C}(0)=\frac{A}{\varrho\left[\bar{b} \delta-\left(b_{1}-b_{2}\right)\right]^{2}}$

No significant density fluctuation takes place upon adding the diluting liquid, as shown in Fig. $5\left(S_{N N}(0)\right.$ remains small). On the other hand, we witness an increase in the concentration fluctuation term $S_{C C}(0)$, which is directly related to the particle clustering in the mixture. This can be explained by the fact that TBA molecules interact preferentially by $\mathrm{H}$-bonds leading to the formation of TBA-rich regions. The typical volume associated to the local variations of the 
solute concentration could be estimated from the correlation length deduced from the OrnsteinZernike analysis. It is striking that it remains close to the molecular scale, which means that the concentration fluctuations roughly scale with the size of the multimeric species characterized by Raman spectroscopy [10].

Another interesting point to check is if the two systems with opposite isotopic compositions i.e. TBA (H)-TOL (D) versus TBA (D)-TOL (H) present the same fluctuation terms $S_{N N}(0)$, $S_{C C}(0)$ and $S_{N C}(0)$ although they exhibit different SANS spectra (cf. Fig. 1). In fact, it is the case as long as isotopic effects on the liquid structure are negligible, which can be safely assumed. The results shown in Fig. 5 confirm that the computed fluctuation terms fulfill this requirement, which validates the method and especially the appropriate correction for the isotopic effects on the values of scattering length densities.

A noticeable solvent effect is worth mentioning when comparing TBA-TOL and TBA-CYC systems, respectively Figs. 5 and 6 . The heterogeneous character of the mixture is prevalent around $x=0.4$ for TBA-TOL systems (highlighted by a maximum in $S_{C C}(0)$ ). This position is shifted to lower composition (about $x=0.3$ ) for the TBA-CYC systems. This observation goes in line with the spectroscopic study mentioned previously, which showed that TBA tetrameric clusters are stable to extreme dilution conditions in aliphatic solvent $(x=0.2)$ and to a lesser extent in TOL because of a competition between TBA-TBA association and TBA-TOL interaction [10]. Again, the phenomenon underlines the link between concentration fluctuations and the existence of multimeric species. 

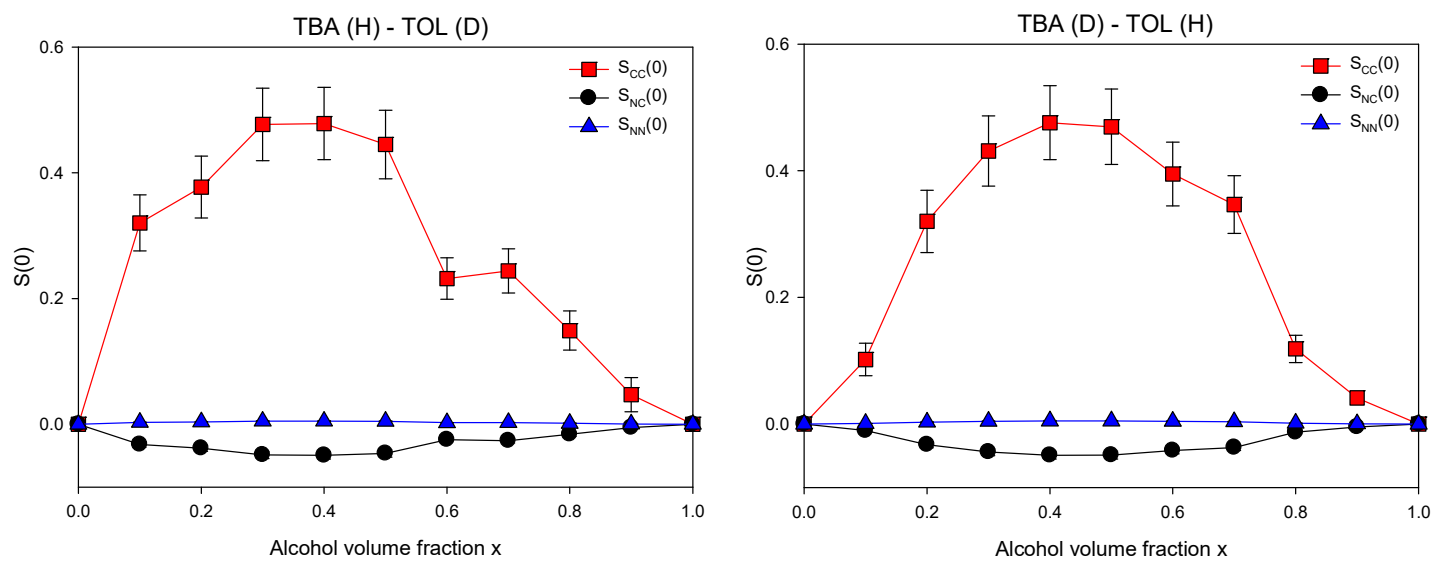

Fig. 5. Bhatia-Thornton fluctuation terms of Tert-butanol-Toluene mixtures, comprising hydrogenated TBA with deuterated TOL (left panel) and vice-versa (right panel). $S_{C C}(0), S_{N N}(0)$ and $S_{N C}(0)$ correspond to concentration fluctuation, density fluctuation and the cross-correlation term, respectively.
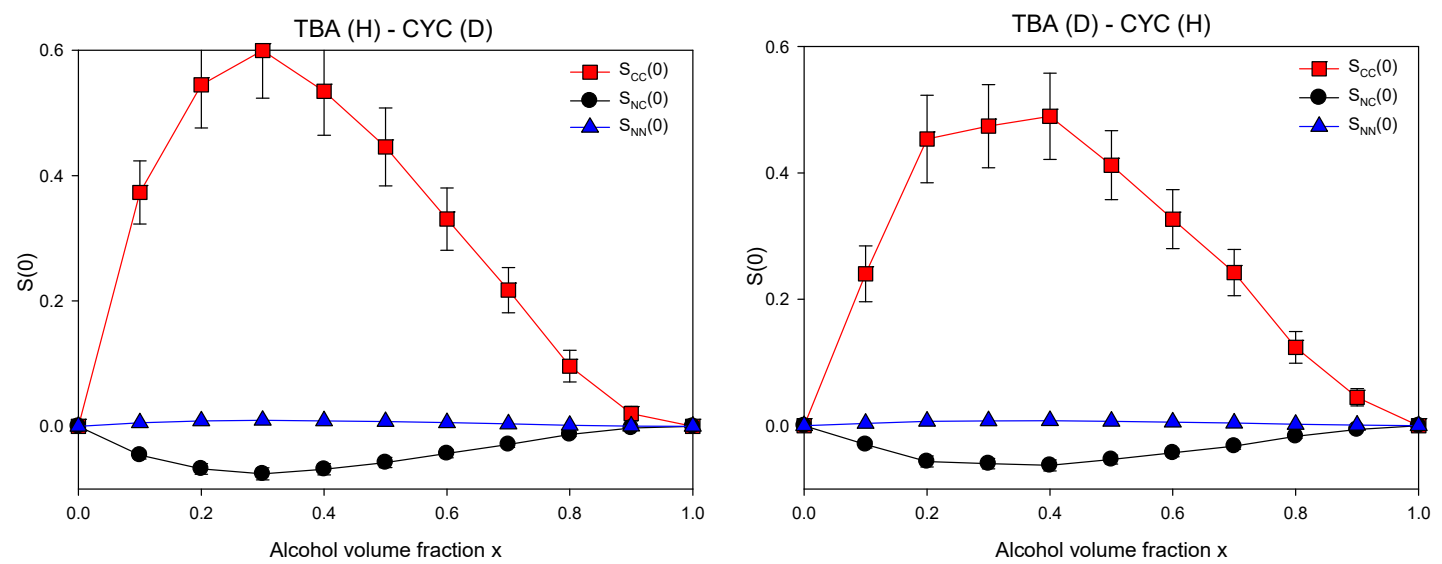

Fig. 6. Bhatia-Thornton fluctuation terms of Tert-butanol-Cyclohexane mixtures, comprising hydrogenated TBA with deuterated CYC (left panel) and vice-versa (right panel). $S_{C C}(0), S_{N N}(0)$ and $S_{N C}(0)$ correspond to concentration fluctuation, density fluctuation and the cross-correlation term, respectively. 


\subsection{Kirkwood-Buff Analysis}

The Kirkwood-Buff theory illustrates the trends of molecular interactions in a solution via the Kirkwood-Buff integrals (KBIs) which can be expressed as [34]:

$$
G_{i j}=\int_{0}^{\infty}\left(g_{i j}(r)-1\right) 4 \pi r^{2} \mathrm{~d} r
$$

It is based on the partial pair correlation functions $\left(g_{i j}(r), g_{i j}(r)\right.$, and $\left.g_{i j}(r)\right)$, which relate the probability of finding a particle at a given distance from another one, appraising the solventsolvent, solute-solvent, and solute-solute interactions. The KBIs provide the tendencies of cluster formation around a given molecule.

SANS is proven to be a suitable technique for a reliable KBIs determination in binary mixtures $[41-43,45]$ where the KBIs can be expressed as linear combination of the different fluctuation terms, where the indices 1 and 2 refer to the aprotic solvent and the alcohol, respectively:

$$
\begin{aligned}
& G_{11}=\frac{1}{\rho}\left(S_{N N}(0)+\frac{2}{x_{1}} S_{N C}(0)+\frac{1}{x_{1}^{2}} S_{C C}(0)-\frac{1}{x_{1}}\right) \\
& G_{22}=\frac{1}{\rho}\left(S_{N N}(0)-\frac{2}{x_{2}} S_{N C}(0)+\frac{1}{x_{2}^{2}} S_{C C}(0)-\frac{1}{x_{2}}\right) \\
& G_{12}=\frac{1}{\rho}\left(S_{N N}(0)+\frac{x_{2}-x_{1}}{x_{1} x_{2}} S_{N C}(0)-\frac{1}{x_{1} x_{2}} S_{C C}(0)\right)
\end{aligned}
$$

The values expected for ideal mixtures can also be computed using the partial volumes as recalled in ref. [46]. We added them to complement the results obtained from SANS measurements in the limit of pure components ( $x=0$ and 1$)$. The Kirkwood Buff integrals shown in Figs. 7 and 8 follow a similar trend in the four different systems. They show a tendency of TBA segregation (which is revealed by an increase in the magnitude of $G_{22}$ at $x<0.4$ ). This segregation of TBA molecules can be linked to a remarkable stability of H-bonded multimeric micellar clusters that are already formed even for very low TBA concentrations through $\mathrm{H}$ bonding. It can be noted that the single value $G_{22} \approx 0$ obtained at $x=0.1$ for the TBA (D)-TOL $(\mathrm{H})$ system stands out of the overall trend. This singular behavior was not observed for any other compositions and so it is likely artefactual. The negligible values of $G_{22}$ at larger $x$ suggest that the interaction between the multimeric H-bonded clusters is much weaker. The two 
Kirkwood Buff integrals containing correlations with the aprotic molecules ( $G_{11}$ and $\left.G_{12}\right)$ remain close to zero, which indicates that the interaction involving TOL or CYC molecules are non-specific.
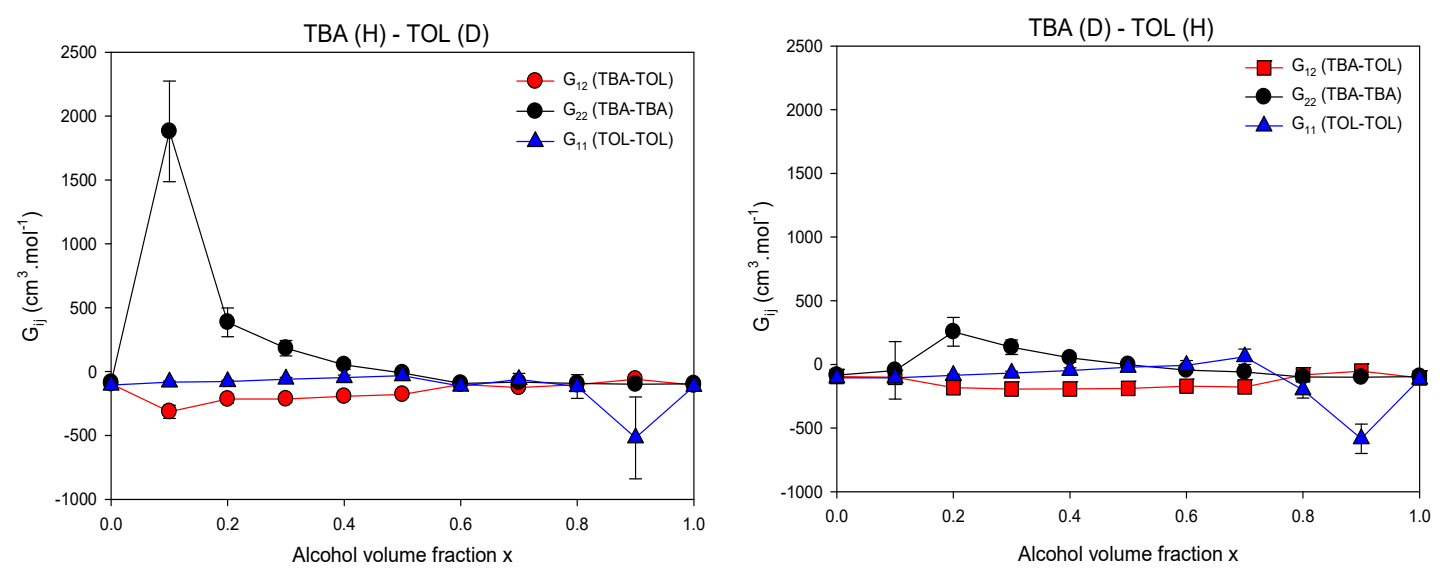

Fig. 7. Kirkwood-Buff integrals of Tert-butanol-Toluene mixtures, comprising hydrogenated TBA with deuterated TOL (left panel) and vice-versa (right panel). $G_{11}$ is the TOL-TOL KB integral, $G_{22}$ is the TBA-TBA $\mathrm{KB}$ integral, and $G_{12}$ is the TBA-TOL KB integral.
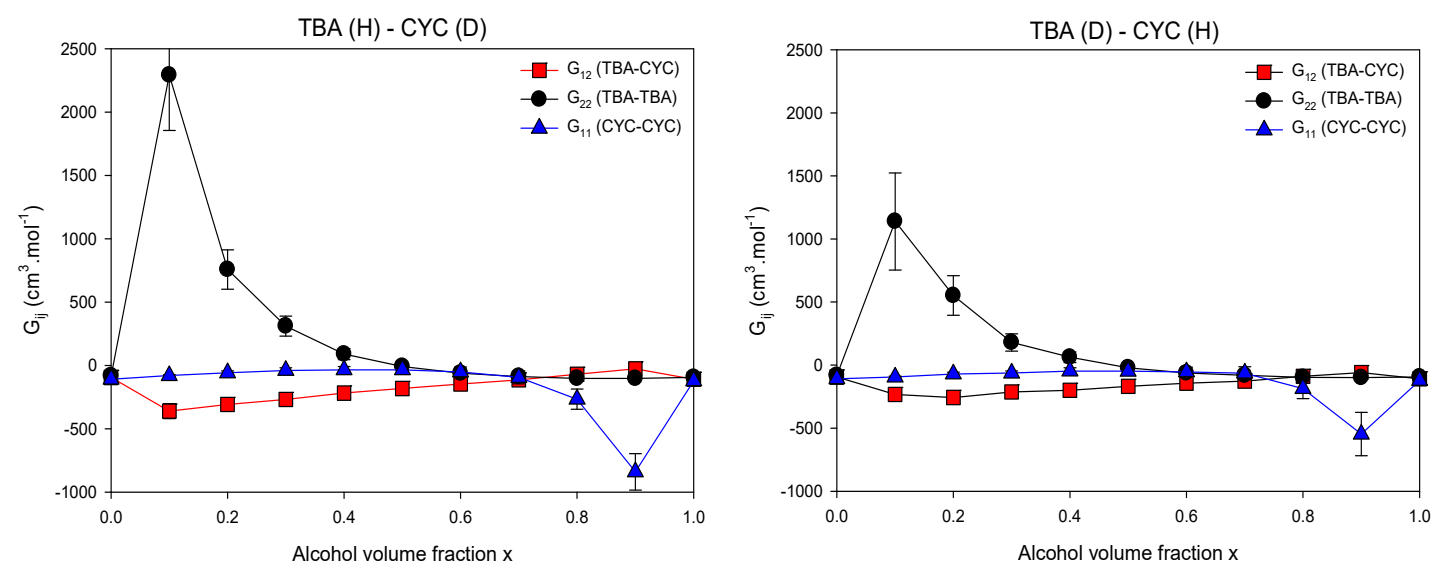

Fig. 8. Kirkwood-Buff integrals of Tert-butanol-Cyclohexane mixtures, comprising hydrogenated TBA with deuterated CYC (left panel) and vice-versa (right panel). $G_{11}$ is the CYC KB integral, $G_{22}$ is the TBA-TBA KB integral, and $G_{12}$ is the TBA-CYC KB integral.

\subsection{Relation between concentration fluctuations, cluster size and prepeak}

In order to gain a more comprehensive description of the different levels of structural organization of the TBA-solvent binary liquids, we have combined the present results to 
existing neutron diffraction and Raman spectroscopy studies [10]. The evolution of the size of the H-bonded micellar clusters deduced by Raman spectroscopy and the diffraction prepeak intensity (at $q=0.7 \AA^{-1}$ ) are compared with the concentration fluctuation term $S_{C C}(0)$ in Fig. 9. For clarity, only the averaged value of $S_{C C}(0)$ on each couple of HD and DH systems is shown. It should be noted that the Raman spectroscopy and large angle neutron diffraction experiments were conducted for fully hydrogenated systems, though no isotopic effect is expected. MethylCyclohexane instead of Cyclohexane was used in these previous experiments, a difference which could have very limited effects on the comparison. Indeed, Methyl-Cyclohexane and Cyclohexane are comparable in molecular size, chemical nature. They have the same value of the dielectric permittivity and unlike benzene derivatives, they do not exhibit specific solvation interaction with TBA $[47,48,49]$.
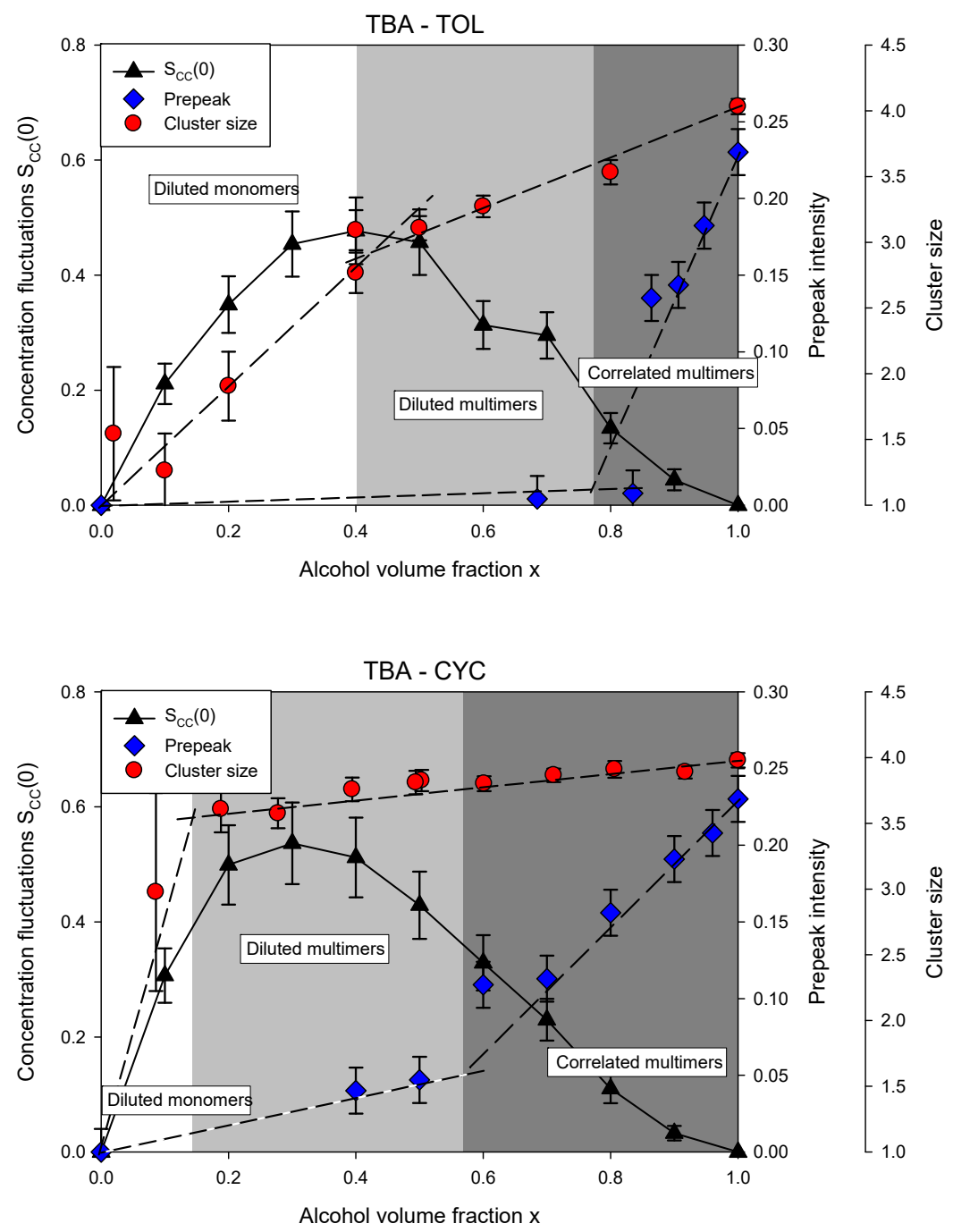

Fig. 9. Bhatia-Thornton fluctuation concentration fluctuation term $S_{C C}(0)$ terms of Tert-butanol-Toluene mixtures (black triangles), compared with the H-bonded cluster size (red circles) and prepeak intensity (blue diamonds) 
adapted from Abdel Hamid et al. [10]. Tert-butanol-Toluene mixtures (upper panel), and Tert-butanolCyclohexane with Tert-butanol-Methylcyclohexane (lower panel).

The distinct evolution of the cluster size and of the prepeak intensity suggested that the formation of H-bonded micellar cluster and the occurrence of interactions between them were independent phenomena. It was suggested the existence of two crossovers separating phases characterized by the predominance of diluted TBA monomers, diluted H-bonded multimeric clusters and TBA-rich domains comprising interacting clusters.

As discussed previously, the low- $q$ scattered intensity measured in the present study by SANS could have different origins, which are related to genuine concentration fluctuations observed in usual molecular binary liquids, the specific microstructures of hydrotropes (i.e. formation of micellar clusters), and the arrangement of domains which contains aggregated TBA clusters likewise the case of microemulsions. The results shown in Fig. 9 demonstrate that the concentration fluctuations increase concomitantly with the formation of micellar clusters, and reach maximum values in the region characterized by the predominance of 'diluted multimers'. A decay of the concentration fluctuations appear for larger concentrations, approaching the region where the aggregation of TBA in mesoscopic domains is indicated by the growth of a prepeak intensity. This confirms that the concentration fluctuations do not arise from mesoscopic domains but from the micellar-clusters themselves, in agreement with the small and constant values of the associated correlation length.

However, it should be pointed out that the values of the correlation length $\xi \approx 2 \AA$ obtained from the Ornstein-Zernike analysis seems too small to provide a consistent microscopic picture. We applied an alternative evaluation, assuming that the small angle scattered intensity involves the form factor of supermolecular units, namely the TBA micellar-clusters. D'Arrigo et al. compared both the Ornstein-Zernike and the Guinier approximation to fit the spectra of micelle-like structures in small-alcohol solutions [32]. They showed that these two alternative ways were numerically equivalent in the low- $q$ region, assuming that the system consists in weakly or non-interacting spherical particles of diameter $D=2 \sqrt{5} \xi$. This result is obtained from a quadratic expansion of the Ornstein-Zernike and Guinier equations giving $R_{g}^{2}=3 \xi^{2}$, and applying the relation between the radius of gyration and the radius of a sphere $R_{g}^{2}=\frac{3}{5} R^{2}$. The assumption that the TBA micellar-clusters do not interact is supported for small alcohol volume fraction $x$ by the absence of prepeak (cf. Fig. 9), which means that the interparticle 
structure factor is close to unity. The application of this relationship for $\xi=2 \AA$ gives $D=8.9 \AA$, which is consistent with the size of the TBA micellar-clusters characterized by Raman and neutron diffraction [10]. Moreover for $x>0.6-0.8$, the prepeak at $q_{P P}=0.7 \AA^{-1}$ arising from of the

correlations between neighboring micellar-clusters allows getting a repeat distance $d=\frac{2 \pi}{q_{P P}}=$ $9 \AA$, which is indeed in very good agreement with the aforementioned particle size.

\section{Ethanol-Toluene and Methanol-Toluene systems}

\subsection{Experimental Results and Ornstein-Zernike Analysis}

Intending to tune the amphiphilic character of the H-bonded molecules, a complementary study has been performed in order to follow a series of alcohol molecules with decreasing alkyl group size: from Tert-butanol (TBA) to Ethanol (ETA) and Methanol (MEA). The comparison between the three alcohols is performed for alcohol-Toluene systems. The SANS spectra of ETA-TOL and MEA-TOL shown in Fig. 10 exhibit an analogous increase in the intensity at low $q$, supplying evidence of aggregation in these solutions too. The parameters obtained from fits with the Ornstein Zernike model of the SANS spectra (lines in Fig. 10) are shown in Fig. 11. Contrary to the TBA systems, the correlation length extracted from the fits varies with the concentration. The correlation length reaches its largest value (5.5 $\AA$ ) for highly diluted alcohol mixtures (being constant in the region $0.1<x<0.3$ ), and then gradually decreases with the increasing quantity of the alcohol. It is also noticeable that the values of the correlation lengths for Methanol and Ethanol are twice as large as for TBA despite their smaller molecular size. The largest values of the correlation lengths are obtained for the smallest alcohol molecule MEA. 

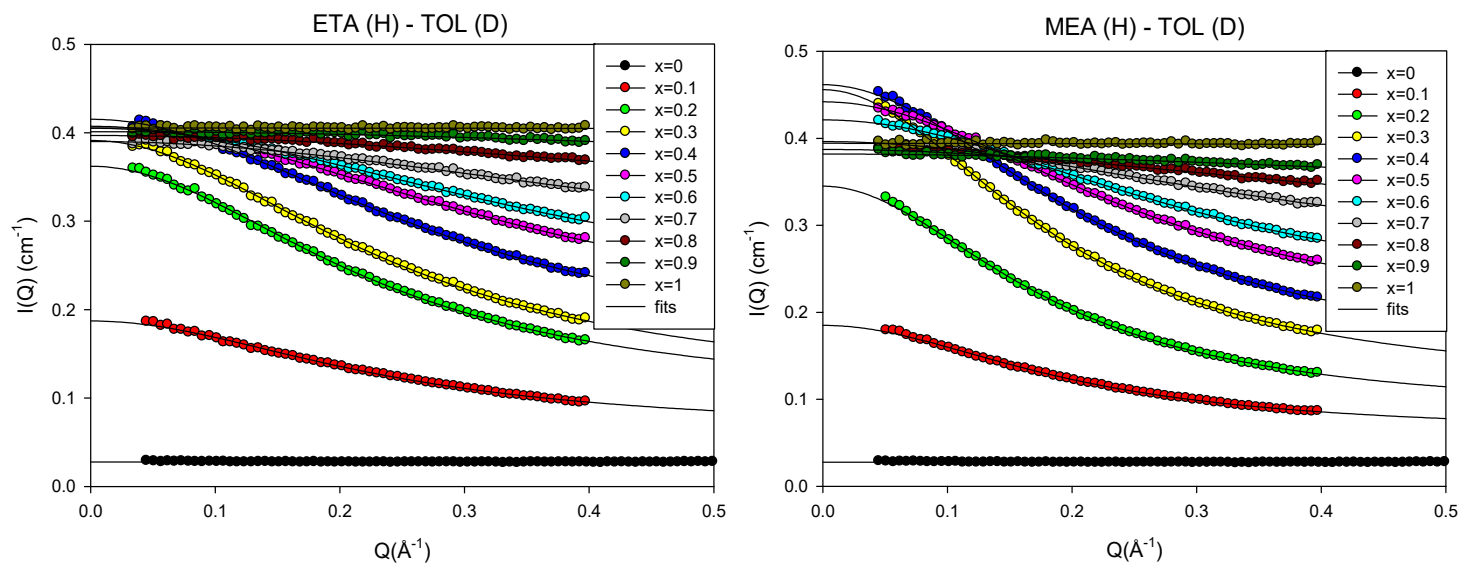

Fig. 10. SANS structure factors of Ethanol-Toluene (left panel) and Methanol-Toluene (right panel) mixtures, comprising hydrogenated alcohol with deuterated TOL. The different curves correspond to eleven values of the alcohol volume fractions $\mathrm{x}$ from 0 to 1 . The plotted solid lines are fits to the Ornstein-Zernike model.
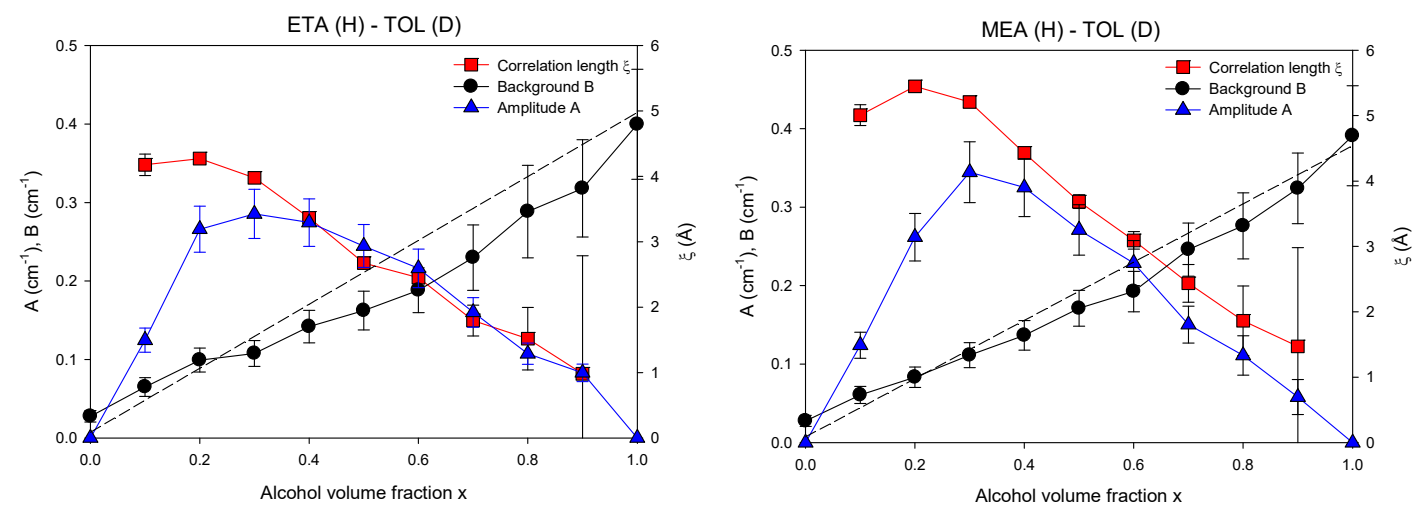

Fig. 11. Parameters (amplitude, correlation length and background) obtained from a fit with the Ornstein-Zernike model of the SANS structure factors of Ethanol-Toluene (left panel) and Methanol-Toluene (right panel) mixtures, comprising hydrogenated alcohol with deuterated TOL.

This different behavior is mostly determined by the balance between hydrophobic and hydrophilic interactions, which depends on the chemical nature of the molecule. A possible interpretation is the formation of extended linear H-bonded clusters in Methanol and Ethanol, while TBA forms cyclic multimers with limited size (typically 4 molecules) $[10,17,22,23$, $24,50,51,52,53,54,55]$. Indeed, experimental and computational studies have reported that Methanol forms mostly chainlike structures comprising up to 10 molecules. They have been related to the prevailing hydrophilic interaction of small alcohol molecules. This situation 
evolves for larger aliphatic group like TBA where the formation of cyclic micellar multimers is promoted by the steric hindrances.

A reason for the variation of the correlation length with the concentration for MEA and ETA could also be proposed. For small alcohol fraction $(0<x<0.3)$, the correlation length reflects the formation of H-bonded alcohol chainlike structures, which are diluted in the TOL solvent. In this region, the correlation length seems rather constant, or might even exhibit a maximum at about $x=0.2$. At variance, on the other side of the graph (i.e. $0.8<x<1$ ), the concentration fluctuations arise from diluted TOL molecules in the alcohol solvent. Because TOL are noninteracting molecules $\left(G_{11} \approx 0\right)$, the associated correlation length is expected to be very small (molecular level). Then, the reduction of the correlation length with increasing alcohol concentration can be understood as the simple continuous behavior connecting these two limit cases.

\subsection{Bhatia-Thornton and Kirkwood-Buff analysis}

The fluctuation terms emphasize the observation of an aggregation process leading to large concentration fluctuation quantified by $S_{C C}(0)$ shown in Fig. 12. It also reveals that the heterogeneous character of the structure of Ethanol-Toluene and Methanol-Toluene solution prevails at lower concentrations (about $x=0.2$ ) than for TBA. To understand this result, it is worth mentioning that the strength of the H-bond interaction increases with decreasing the size of the alkyl part of the alcohol, which enhances the stabilization of H-bonded multimers of TEA and MEA diluted in Toluene. Hence, the systematic evolution of $S_{C C}(0)$ with the selfassociation tendency going from TBA, ETA to MEA supports the idea that concentration fluctuations are dominated by the formation of the multimeric clusters themselves.

Moreover a change in the density-density fluctuation $S_{N N}(0)$ is also witnessed in high dilution conditions following the same trend as $S_{C C}(0)$. This observation can be easily interpreted as the coupling between concentration fluctuations and density fluctuations for binary mixtures with constituents having different molar volumes (i.e. large dilatation factor $\delta$ according to Eq. (4)). This phenomenon was much weaker for TBA-TOL mixture, both components having comparable molar volumes so that their density did not depend significantly on the concentration.

The Kirkwood Buff theory also goes well with the precedent analysis as it shows in Fig. 13 the increase of $G_{22}$ at low alcohol concentrations, which demonstrates strong alcohol- 
alcohol interactions. The KBI remain very small on the alcohol-rich side because of weaker van-der-Waals TOL-TOL interactions [56].
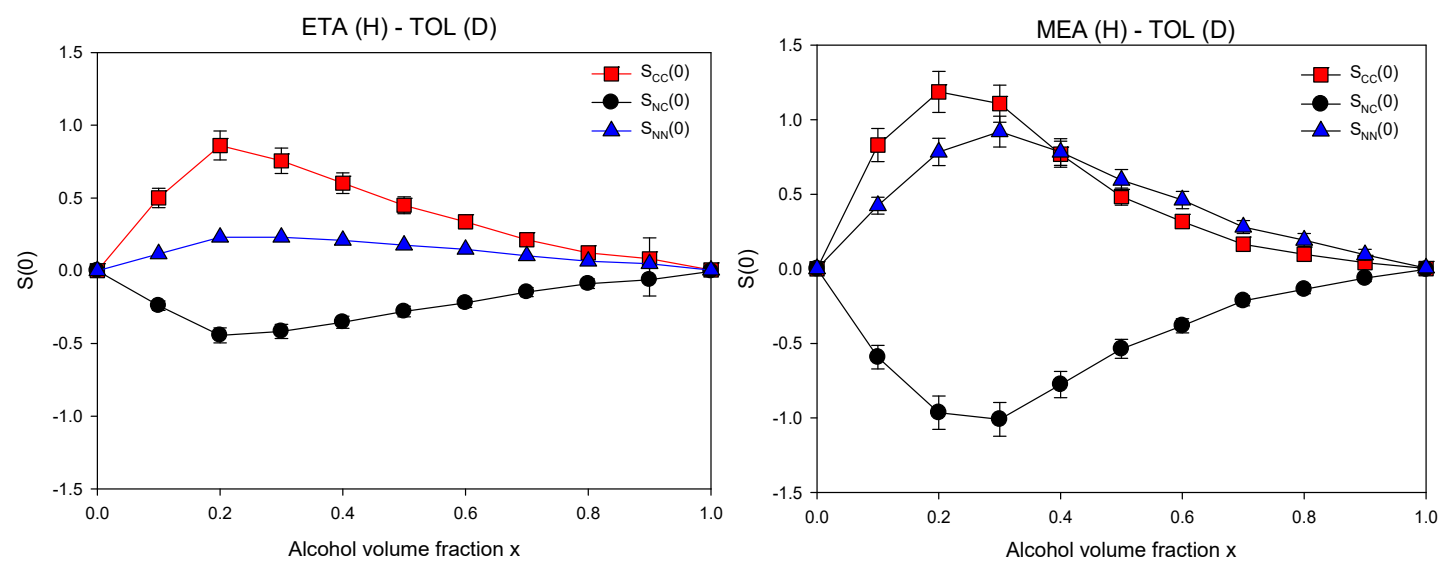

Fig. 12. Bhatia-Thornton fluctuation terms of Ethanol-Toluene (left panel) and Methanol-Toluene (right panel) mixtures, comprising hydrogenated alcohol with deuterated TOL. $S_{C C}(0), S_{N N}(0)$ and $S_{N C}(0)$ correspond to concentration fluctuation, density fluctuation and the cross-correlation term, respectively.
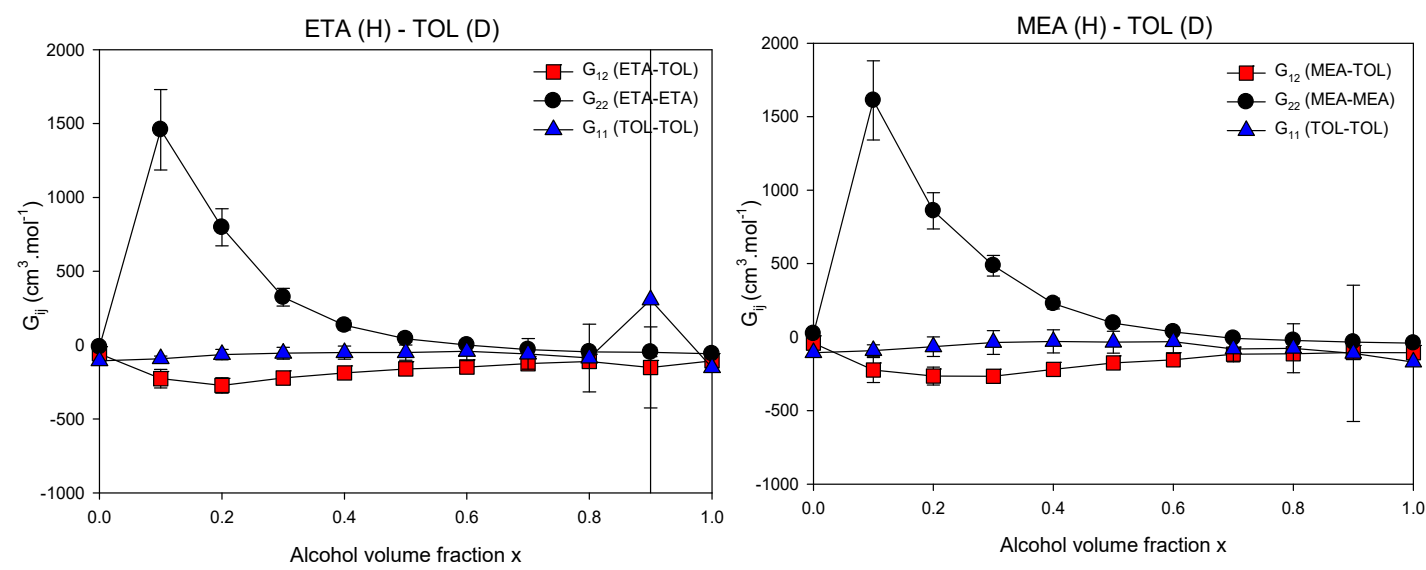

Fig. 13. Kirkwood-Buff integrals of Ethanol-Toluene (left panel) and Methanol-Toluene (right panel) mixtures, comprising hydrogenated alcohol with deuterated TOL. $G_{11}$ is the TOL KB integral, $G_{22}$ is the alcohol-Alcohol $\mathrm{KB}$ integral, and $G_{12}$ is the Alcohol-TOL KB integral.

\section{Summary and conclusion}

It is a long-established agreement that alcohol binary liquids are not homogeneous but exhibit peculiar molecular organizations. Going further in detail, it turns out that the structural 
complexity of such systems reflects the interplay between different parameters related to the $\mathrm{H}$-bond association itself, the self-aggregation with formation of $\mathrm{H}$-bonded micellar clusters and the mesoscopic correlations or concentration fluctuations; different aspects that has been assessed in the present study.

The present study has been performed in the context of many debated issues about the solvation of hydrotropes, highlighted by studies on small-chains alcohols in water. The introduction of the concept of molecular emulsions and the application of the Teubner and Strey models to assess the original structure of TBA-water solutions, raises questions about the inter-clusters correlations of TBA in other hydrophobic aprotic solvents. According to recent studies, the microstructure of pure TBA and TBA in aprotic solvents is characterized by $\mathrm{H}-$ bonded micellar clusters, comprising typically four H-bonded molecules, which remain stable during dilution on the broad range of concentrations, as large as $x=0.1-1$ for TBA-MCYC. The cyclic structure of TBA multimers in the pure component resembles reverse-micelles, which can explain their larger stability in hydrophobic solvent (oil) than in water. Mesoscopic correlations between these clusters are indicated by a prepeak, but it is very sensitive to dilution and rapidly decays. This indicates that the microstructure of TBA-aprotic solvents mixtures could be rather expressed in terms of uncorrelated micellar clusters, at least for a broad range of intermediate compositions.

The SANS experiments performed for a series of TBA-CYC and TBA-TOL mixtures with different concentrations and isotopic compositions exhibit a systematic increase of the scattered intensity at low- $q$. This feature having no connection with the prepeak nor any other diffraction peak, this asymptotic behavior is more likely related to concentration fluctuations. The characteristic correlation length of the inhomogeneities has been determined using the Ornstein-Zernike model. Its value remains close to the molecular size itself, and importantly it appears independent on the existence or non-existence of intercluster correlations (i.e. prepeak). This suggests that the low- $q$ intensity could be alternatively modelled by the form factor of well-defined supermolecular units. Assuming a spherical shape, this evaluation provided a value of the diameter $D=8.9 \AA$, which is in good agreement with the size of the TBA H-bonded micellar clusters deduced from the prepeak repeat distance $d=\frac{2 \pi}{q_{P P}}=9 \AA$, corresponding to the interclusters interaction distance at much larger TBA fraction. 
The separation between density and concentration correlation functions has been performed by application of the formalism of Bhatia and Thornton at $q=0$. The evolution of the concentration fluctuation $S_{C C}(0)$ was compared with published data on the H-bonded cluster size and the prepeak intensity. The behaviors of both the Ornstein-Zernike correlation length and Bhatia and Thornton concentration fluctuation $S_{C C}(0)$ support that concentration fluctuations do not arise from fluctuations of the size and arrangement of the domains aggregating TBA micellar clusters but from the micellar-clusters themselves. The increase of the Kirwood-Buff integrals for very low TBA concentration, underlines that the microstructure of TBA is driven by strong H-bond interactions and the spectacular stability of micellar clusters, while their tendency to aggregate into TBA rich domains is related to much weaker interactions.

While these conclusions seem to apply generally for smaller alcohol molecules too, such as Ethanol and Methanol, systematic variations are observed. The smaller the size of the alcohol molecule is, the larger the values of the correlation lengths. This effect could be related to the different microstructures, more often expressed in terms of extended linear H-bonded clusters in Methanol and Ethanol, while TBA is known to form cyclic multimers with limited sizes (typically 4 molecules). Beyond the interests for organic solvent properties, the mesoscopic approach provided by the SANS study on alcohol-aprotic solvent mixtures could bring new experimental views on issues currently debated for aqueous-alcohols mixtures about the relation between concentration fluctuations and supramolecular micellar clusters $[5,8,16,57]$.

\section{Acknowledgement}

This study was part of the $\mathrm{PhD}$ project of $\mathrm{R}$. Mhanna, co-funded by the Institut LaueLangevin and the Brittany Region (ARED 7784 / NanoBina). Support from Europe (FEDER) and Rennes Metropole is expressly acknowledged.

\section{References}

1. Dixit, S.; Crain, J.; Poon, W. C. K.; Finney, J. L.; Soper, A. K. Molecular segregation observed in a concentrated alcohol-water solution. Nature 2002, 416 (6883), 829-832. 
2. Guo, J. H.; Luo, Y.; Augustsson, A.; Kashtanov, S.; Rubensson, J. E.; Shuh, D. K.; Agren, H.; Nordgren, J. Molecular structure of alcohol-water mixtures. Physical Review Letters 2003, $91(15)$.

3. Wakisaka, A.; Matsuura, K. Microheterogeneity of ethanol-water binary mixtures observed at the cluster level. Journal of Molecular Liquids 2006, 129 (1-2), 25-32.

4. Dougan, L.; Crain, J.; Finney, J. L.; Soper, A. K. Molecular self-assembly in a model amphiphile system. Physical Chemistry Chemical Physics 2010, 12 (35), 10221-10229.

5. Subramanian, D.; Boughter, C. T.; Klauda, J. B.; Hammouda, B.; Anisimov, M. A. Mesoscale inhomogeneities in aqueous solutions of small amphiphilic molecules. Faraday Discussions 2013, 167, 217-238.

6. Kezic, B.; Perera, A. Revisiting aqueous-acetone mixtures through the concept of molecular emulsions. Journal of Chemical Physics 2012, 137 (13).

7. Perera, A.; Mazighi, R.; Kezic, B. Fluctuations and micro-heterogeneity in aqueous mixtures. Journal of Chemical Physics 2012, 136 (17).

8. Perera, A.; Kezic, B. Fluctuations and micro-heterogeneity in mixtures of complex liquids. Faraday Discussions 2013, 167, 145-158.

9. Kezic, B.; Perera, A. Aqueous tert-butanol mixtures: A model for molecular-emulsions. Journal of Chemical Physics 2012, 137 (1).

10. Abdel Hamid, A.; Lefort, R.; Lechaux, Y.; Moréac, A.; Ghoufi, A.; Alba-Simionesco, C.; Morineau, D. Solvation Effects on Self-Association and Segregation Processes in tertButanol-Aprotic Solvent Binary Mixtures. The Journal of Physical Chemistry B 2013, 117 (35), 10221-10230.

11. Hennous, L.; Hamid, A.; Lefort, R.; Morineau, D.; Malfreyt, P.; Ghoufi, A. Crossover in structure and dynamics of a primary alcohol induced by hydrogen-bonds dilution. Journal of Chemical Physics 2014, 141 (20).

12. Wang, L. M.; Shahriari, S.; Richert, R. Diluent effects on the Debye-type dielectric relaxation in viscous monohydroxy alcohols. Journal of Physical Chemistry B 2005, 109 (49), $23255-23262$.

13. El Goresy, T.; Bohmer, R. Diluting the hydrogen bonds in viscous solutions of nbutanol with n-bromobutane: A dielectric study. Journal of Chemical Physics 2008, 128 (15). 14. Lederle, C.; Hiller, W.; Gainaru, C.; Bohmer, R. Diluting the hydrogen bonds in viscous solutions of n-butanol with n-bromobutane: II. A comparison of rotational and translational motions. Journal of Chemical Physics 2011, 134 (6). 
15. Artola, P. A.; Raihane, A.; Crauste-Thibierge, C.; Merlet, D.; Emo, M.; AlbaSimionesco, C.; Rousseau, B. Limit of Miscibility and Nanophase Separation in Associated Mixtures. Journal of Physical Chemistry B 2013, 117 (33), 9718-9727.

16. Wilcox, D. S.; Rankin, B. M.; Ben-Amotz, D. Distinguishing aggregation from random mixing in aqueous t-butyl alcohol solutions. Faraday Discussions 2013, 167, 177-190.

17. Subramanian, D.; Klauda, J. B.; Collings, P. J.; Anisimov, M. A. Mesoscale Phenomena in Ternary Solutions of Tertiary Butyl Alcohol, Water, and Propylene Oxide. Journal of Physical Chemistry B 2014, 118 (22), 5994-6006.

18. Rankin, B. M.; Ben-Amotz, D.; van der Post, S. T.; Bakker, H. J. Contacts Between Alcohols in Water Are Random Rather than Hydrophobic. Journal of Physical Chemistry Letters 2015, 6 (4), 688-692.

19. Comez, L.; Paoantoni, M.; Lupi, L.; Sassi, P.; Corezzi, S.; Morres, A.; Fioretto, D. Hydrophobic Hydration in Water-tert-Butyl Alcohol Solutions by Extended Depolarized Light Scattering. Journal of Physical Chemistry B 2015, 119 (29), 9236-9243.

20. Palombo, F.; Paolantoni, M.; Sassi, P.; Morresi, A.; Cataliotti, R. S. Spectroscopic studies of the "free" OH stretching bands in liquid alcohols. Journal of Molecular Liquids 2006, 125 (2-3), 139-146.

21. Sassi, P.; Palombo, F.; Cataliotti, R. S.; Paolantoni, M.; Morresi, A. Distributions of Hbonding aggregates in tert-butyl alcohol: The pure liquid and its alkane mixtures. Journal of Physical Chemistry A 2007, 111 (27), 6020-6027.

22. Kusalik, P. G.; Lyubartsev, A. P.; Bergman, D. L.; Laaksonen, A. Computer simulation study of tert-butyl alcohol. 1. Structure in the pure liquid. Journal of Physical Chemistry B 2000, 104 (40), 9526-9532.

23. Perera, A.; Sokolic, F.; Zoranic, L. Microstructure of neat alcohols. Physical Review E 2007, 75 (6).

24. Zoranic, L.; Sokolic, F.; Perera, A. Microstructure of neat alcohols: A molecular dynamics study. Journal of Chemical Physics 2007, 127 (2).

25. Ghoufi, A.; Hureau, I.; Lefort, R.; Morineau, D. Hydrogen-Bond-Induced Supermolecular Assemblies in a Nanoconfined Tertiary Alcohol. Journal of Physical Chemistry C 2011, 115 (36), 17761-17767.

26. Ghoufi, A.; Hureau, I.; Morineau, D.; Renou, R.; Szymczyk, A. Confinement of tertButanol Nanoclusters in Hydrophilic and Hydrophobic Silica Nanopores. Journal of Physical Chemistry C 2013, 117 (29), 15203-15212. 
27. Morineau, D.; Alba-Simionesco, C. Does Molecular Self-Association Survive in Nanochannels? Journal of Physical Chemistry Letters 2010, 1 (7), 1155-1159.

28. Morineau, D.; Alba-Simionesco, C.; Bellissent-Funel, M.; Lauthie, M. Experimental indication of structural heterogeneities in fragile hydrogen-bonded liquids. EUROPHYSICS LETTERS 1998, 43 (2), 195-200.

29. Morineau, D.; Alba-Simionesco, C. Hydrogen-bond-induced clustering in the fragile glass-forming liquid m-toluidine: Experiments and simulations. Journal of Chemical Physics 1998, 109 (19), 8494-8503.

30. Alba-Simionesco, C.; Morineau, D.; Frick, B.; Higonenq, N.; Fujimori, H. An analysis of the short and intermediate range order in several organic glass-forming liquids from the static structure factor under pressure. Journal of Non-Crystalline Solids 1998, 235, 367-374.

31. Debye, P.; Bueche, A. M. SCATTERING BY AN INHOMOGENEOUS SOLID. Journal of Applied Physics 1949, 20 (6), 518-525.

32. Darrigo, G.; Teixeira, J. SMALL-ANGLE NEUTRON-SCATTERING STUDY OF D2O ALCOHOL-SOLUTIONS. Journal of the Chemical Society-Faraday Transactions 1990, $86(9), 1503-1509$.

33. Bhatia, A. B.; Thornton, D. E. Structural aspects of the electrical resistivity of binary alloys. Physical Review B-Solid State 1970, 2 (8), 3004-3012.

34. Kirkwood, J. G.; Buff, F. P. The statistical mechanical theory of solutions. I. The Journal of chemical physics 1951, 19 (6), 774-777.

35. Teubner, M.; Strey, R. ORIGIN OF THE SCATTERING PEAK IN MICROEMULSIONS. Journal of Chemical Physics 1987, 87 (5), 3195-3200.

36. D'Arrigo, G.; Giordano, R.; Teixeira, J. Small-angle neutron scattering studies of aqueous solutions of short-chain amphiphiles. European Physical Journal E 2003, 10 (2), 135 142.

37. Diat, O.; Klossek, M. L.; Touraud, D.; Deme, B.; Grillo, I.; Kunz, W.; Zemb, T. Octanol-rich and water-rich domains in dynamic equilibrium in the pre-ouzo region of ternary systems containing a hydrotrope. Journal of Applied Crystallography 2013, 46, 1665-1669.

38. Stanley, H. E. Introduction to phase transitions and critical phenomena. Introduction to Phase Transitions and Critical Phenomena, by H Eugene Stanley, pp. 336. Foreword by H Eugene Stanley. Oxford University Press, Jul 1987. ISBN-10: 0195053168. ISBN-13: $97801950531661987,1$.

39. Chieux, P.; Damay, P. LONG WAVELENGTH THERMODYNAMIC LIMIT OF A NEUTRON-DIFFRACTION EXPERIMENT IN VICINITY OF A LIQUID-LIQUID 
CRITICAL-POINT - APPLICATION TO CONCENTRATION FLUCTUATIONS IN LIND3 SYSTEM. Chemical Physics Letters 1978, 58 (4), 619-621.

40. Coulet, M.-V.; Bellissent, R.; Bichara, C. Small-angle neutron scattering study of sulphur-tellurium liquid alloys: structural evidence for a phase separation. Physica B: Condensed Matter 2000, 276, 415-416.

41. Almasy, L.; Jancso, G. Small-angle neutron scattering and Kirkwood-Buff integral study of aqueous solutions of pyridine. Journal of molecular liquids 2004, 113 (1-3), 61-66.

42. Almásy, L.; Cser, L.; Jancsó, G. SANS study of 3-methylpyridine-heavy water mixtures. Physica B: Condensed Matter 2000, 276, 446-447.

43. Almásy, L.; Jancsó, G.; Cser, L. Application of SANS to the determination of Kirkwood-Buff integrals in liquid mixtures. Applied Physics A 2002, 74 (1), s1376-s1378.

44. Pena, M. P.; Martinez-Soria, V.; Monton, J. B. Densities, refractive indices, and derived excess properties of the binary systems tert-butyl alcohol plus toluene, plus methylcyclohexane, and plus isooctane and toluene plus methylcyclohexane, and the ternary system tert-butyl alcohol plus toluene plus methylcyclohexane at 298.15 K. Fluid Phase Equilibria 1999, $166(1), 53-65$.

45. Shulgin, I.; Ruckenstein, E. Kirkwood-Buff integrals in aqueous alcohol systems: Comparison between thermodynamic calculations and X-ray scattering experiments. The Journal of Physical Chemistry B 1999, 103 (13), 2496-2503.

46. Pozar, M.; Seguier, J.-B.; Guerche, J.; Mazighi, R.; Zoranic, L.; Mijakovic, M.; KezicLovrincevic, B.; Sokolic, F.; Perera, A. Simple and complex disorder in binary mixtures with benzene as a common solvent. Physical Chemistry Chemical Physics 2015, 17 (15), 98859898.

47. Maryott, A., A.; R., S. E. Table of Dielectric Constants of Pure Liquids. 1951; Vol. 514.

48. Larsen, G.; Ismail, Z. K.; Herreros, B.; Parra, R. D. Benzene tert-butyl alcohol interactions. 1. A theoretical and experimental study. Journal of Physical Chemistry A 1998, $102(24), 4734-4741$.

49. Larsen, G.; Ismail, Z. K. Monomeric tert-butanol in benzene and cyclohexane solutions: A Fourier-transform infrared spectroscopy study. Journal of Solution Chemistry 1998, 27 (10), 901-909.

50. Adya, A. K.; Bianchi, L.; Wormald, C. J. The structure of liquid methanol by H/D substitution technique of neutron diffraction. Journal of Chemical Physics 2000, 112 (9), 42314241. 
51. Yamaguchi, T.; Hidaka, K.; Soper, A. K. The structure of liquid methanol revisited: A neutron diffraction experiment at -80 degrees $\mathrm{C}$ and +25 degrees C. Molecular Physics 1999, $96(8), 1159-1168$.

52. Kosztolanyi, T.; Bako, I.; Palinkas, G. Hydrogen bonding in liquid methanol, methylamine, and methanethiol studied by molecular-dynamics simulations. Journal of Chemical Physics 2003, 118 (10), 4546-4555.

53. Morineau, D.; Guegan, R.; Xia, Y.; Alba-Simionesco, C. Structure of liquid and glassy methanol confined in cylindrical pores. Journal of Chemical Physics 2004, 121 (3), 1466-1473. 54. Guégan, R.; Morineau, D.; Alba-Simionesco, C. Interfacial structure of an H-bonding liquid confined into silica nanopore with surface silanols. Chemical Physics 2005, 317 (2-3), 236-244.

55. Bowron, D. T.; Finney, J. L.; Soper, A. K. The structure of pure tertiary butanol. Molecular Physics 1998, 93 (4), 531-543.

56. Morineau, D.; Dosseh, G.; Pellenq, R.; Bellissent-Funel, M.; Alba-Simionesco, C. Thermodynamic and structural properties of fragile glass-forming toluene and meta-xylene: Experiments and Monte-Carlo simulations. MOLECULAR SIMULATION 1997, 20 (1-2), 95113.

57. General discussion. Faraday Discussions 2013, 167, 239-262. 